\title{
Propuesta de un Programa Virtual en Bibliotecología y Estudios de la Información para la Universidad de La Salle, Bogotá-Colombia
}

\author{
Johann Enrique Pirela Morillo* \\ Luis Fernando Sierra Escobar* \\ Yamely Almarza Franco*
}

Artículo recibido:

6 de mayo de 2021

Artículo aceptado:

13 de septiembre de 2021

Artículo de investigación

\section{Resumen}

El objetivo de este artículo es diseñar la propuesta de un Programa Virtual en Bibliotecología y Estudios de la Información para la Universidad de La Salle de Bogotá-Colombia. Dicha propuesta se generó como resultado de un proceso de investigación-innovación curricular, realizado para estar en sintonía con las tendencias actuales que se plantean a la disciplina y profesión bibliotecológica y, de modo particular, las propuestas relacionadas con los procesos formativos en este campo de conocimiento. La metodología consideró la triangulación de fuentes e información que se derivó de la aplicación de diferentes técnicas de investigación: comparación de programas académicos similares, encuesta

* Universidad de La Salle, Bogotá-Colombia

jepirela@unisalle.edu.co lusierra@lasalle.edu.co yalmarza@unisalle.edu.co

INVESTIGACIÓN BIBLIOTECOLÓGICA, vol. 36, núm. 90, enero/marzo, 2022, México, ISSN: 2448-8321 pp. 67-85 
realizada a una muestra intencional-focalizada y lineamientos señalados por el Ministerio de Educación $\mathrm{Na}$ cional de Colombia y la Universidad de La Salle para los programas virtuales. Las conclusiones plantean la necesidad de ofrecer programas académicos en este campo de conocimiento y bajo la modalidad virtual como una estrategia de ampliación de cobertura educativa y de inclusión socioformativa, que logre la generación de alternativas de educación bibliotecológica que se requieren no sólo en Colombia, sino en América Latina.

Palabras clave: Educación Bibliotecológica; Formación de Profesionales de la Información; Programas Académicos Virtuales

Proposal for a Virtual Program in Library Science and Information Studies for the University of La Salle, Bogotá-Colombia

Johann Enrique Pirela Morillo, Luis Fernando Sierra Escobar and Yamely Almarza Franco

\section{Abstract}

The general objective of this article is to present the proposal of a Virtual Program in Library Science and Information Studies for the Universidad de La Salle in Bogotá-Colombia. This proposal was generated as a result of a curricular research-innovation process, carried out to be in tune with the current trends that arise in the library discipline and profession and, in a particular way, the proposals related to the training processes in this field of education. knowledge. The methodology considered the triangulation of sources and information that was derived from the application of different research techniques: comparison of similar academic programs, survey carried out on an intentional-focused sample and guidelines indicated by the Ministry of National Education of Colombia and the University of La Salle for virtual programs. The conclusions raise the need to offer academic programs in this field of knowledge and under the virtual modality, as a strategy to expand educational coverage and socio-formative inclusion, for achieving the generation of library education alternatives that are required not only in Colombia, but in Latin America.

Keywords: Library Education; Information Professional Training; Virtual Academic Programs 


\section{INTRODUCCIÓN}

— 1 artículo presenta los productos de un proceso de investigación instiEtucional, desarrollado desde el Programa de Sistemas de Información, Bibliotecología y Archivística de la Universidad de La Salle. Tales productos son el perfil profesional expresado en competencias, la estructura de la malla curricular y la matriz de resultados de aprendizaje. La investigación se planteó desde la innovación curricular para diseñar un Programa Virtual en Bibliotecología y Estudios de la Información y con ocho semestres de duración, considerando las transformaciones académicas, curriculares y formativas que se adelantan en la Universidad de La Salle (Bogotá-Colombia), basadas en el reconocimiento de los entornos globales y las complejidades surgidas en las sociedades de la información y el conocimiento que imponen desafíos a las universidades, sobre todo aquellas que cuentan con capital intelectual para ofrecer programas de bibliotecología y estudios de la información, como es el caso de la Universidad de La Salle, institución de educación superior en la que se fundó el primer programa académico en este campo de conocimiento en la capital de la república colombiana. La experiencia acumulada durante 50 años en educación bibliotecológica y archivística permitió construir un importante capital académico que se constituye en uno de los insumos fundamentales para diseñar la propuesta de programa virtual que se presenta en este estudio.

La sociedad actual y las urgentes necesidades del desarrollo científico demandan nuevos programas estructurados con innovación, creatividad y pensamiento estratégico, lo que amerita la conformación de nuevas capacidades institucionales para responder a las exigencias de las disciplinas, la interconexión entre éstas, los diálogos con los sectores socio-productivos y la consideración de los avances tecnológicos que han servido de plataformas para la producción de conocimiento científico, el cual debe fluir desde los espacios de construcción hacia la sociedad como una forma de validación y contrastación, pero también como un modo de traducir los descubrimientos científicos en productos y acciones que transformen los escenarios sociales y productivos.

Así, la formación en bibliotecología debe volver la mirada hacia aspectos abordados en periodos anteriores, pero ahora con nuevos matices, al colocar a la información científica en el epicentro de los objetos de estudio de este campo de conocimiento, históricamente vinculados con la organización de la información documental y la provisión de servicios bibliotecarios, de apoyo a los procesos educativos, investigativos y de emprendimiento. Los referentes que justifican un programa profesional con esta orientación se ubican, en primer lugar, en la importancia de la noción de ciencia abierta, en términos 
de lo planteado por Anglada y Abadal (2018) sobre la base del modelo de las revoluciones científicas, señalado por Khun. Siguiendo esta idea, la ciencia abierta implica un cambio de paradigma en los modos de llevar a cabo la investigación y en las maneras mediante las cuales los actores/as se apropian de conceptos y prácticas científicas para transformar la realidad.

Los mencionados autores consideran que la ciencia abierta implica nuevas miradas frente al proceso de investigación que da origen a la generación de información científica. Uno de los elementos del movimiento de ciencia abierta es el acceso sin restricciones a los contenidos académicos. En el contexto de este movimiento, lo que debe estar en abierto es cualquier resultado de la investigación (artículos y datos) así como los instrumentos auxiliares usados, como pueden ser los cuadernos de laboratorio. Del mismo modo que lo era para artículos, aquí el significado de "abierto" es doble: gratuito y libre; de manera que se construye también la idea de una ciencia hecha para y con la sociedad, basada además en un estudio sistemático de las necesidades de conocimiento científico para la superación de los problemas sociales. De este modo, el Programa Virtual en Bibliotecología y Estudios de la Información se percibe desde las potencialidades de contar con el talento humano altamente calificado y con una elevada sensibilidad social para conectar ecosistemas de producción de conocimiento científico con las necesidades de la sociedad en su conjunto.

Los componentes de la ciencia abierta señalados por Unesco (2021), en los cuales puede participar el profesional en bibliotecología y estudios de la información, añadiendo valor con la definición de estrategias concretas, son los siguientes: acceso abierto, datos abiertos, código abierto/software libre y hardware abierto, infraestructuras de la ciencia abierta, evaluación abierta, recursos educativos abiertos, participación abierta de los agentes sociales, y apertura a la diversidad de los conocimientos. Así, la ciencia abierta ofrece beneficio colectivo, equidad, justicia, calidad, integridad y diversidad.

Además de la ciencia abierta, se plantean otros retos a la formación bibliotecológica y de estudios de la información relacionados con las humanidades digitales y la gestión de datos en general, que implican competencias renovadas para llevar adelante acciones sistemáticas que pueden desarrollar los profesionales de bibliotecología y estudios de la información, en diálogo con otros profesionales, como los de cómputo, ciencias sociales y humanas, pues no sólo tendrán que desarrollar competencias para intervenir el conocimiento ya generado en los procesos de edición y organizaciónsistematización, sino también la participación y el aporte de la bibliotecología que puede evidenciarse desde los procesos iniciales de creación de contenidos científicos. De modo que se está en presencia de una nueva bibliotecología 
que participe de una forma más decisiva en el análisis de datos, el acceso a la información cultural y científica y la formación de capacidades en los usuarios para interactuar eficazmente en los ecosistemas digitales.

\section{Referentes de la propuesta de Programa Virtual en Bibliotecología y Estudios de la InFormación}

El escenario que plantean la ciencia abierta, las humanidades digitales y el análisis masivo de datos se muestra como oportunidades para la formación de profesionales de bibliotecología y estudios de la información, por constituirse en vías que colocan a disposición el aprender de las buenas prácticas para aportar a los problemas relacionados con la información y la comunicación que la sociedad pueda estar presentando y que a través de proyectos de transformación digital requieren del aporte de profesionales especializados en organización de la información. De esta manera, estimula a que otros puedan colaborar y contribuir cuando los datos de investigación, notas de laboratorio y otros procesos de investigación son de acceso abierto y con términos que permitan la reutilización, la redistribución y la reproducción de la investigación (Rozemblum, 2019).

En este marco, los entornos de aprendizaje virtual se potencian para la generación de nuevos conocimientos desde el trabajo colaborativo y para trascender las fronteras disciplinares que relacionen procesos, contenidos curriculares, recursos u objetos virtuales de aprendizaje (OVA) a través del modelamiento virtual. Para que tal apuesta sea exitosa, es necesario tomar en consideración el potencial cognitivo de los estudiantes, sus competencias tecnológicas, informacionales y comunicativas. En palabras de Merchán (2018), en el modelamiento de ambientes virtuales de aprendizaje (AVA) se articulan cuatro componentes (tecnológico, pedagógico, comunicativo, cognitivo) con el fin de facilitar los procesos de aprendizaje que el aprendiz realiza al usar el ambiente virtual y alcanzar así la intencionalidad.

Es importante también considerar las diversas opciones de plataformas existentes para el aprendizaje virtual, que demuestran su calidad en las posibilidades de interacción y eficacia para el aprendizaje (Morales Salas, Infante Moro y Gallardo Pérez, 2019). Las percepciones sobre la influencia de los estilos de aprendizaje en los entornos virtuales han permitido identificar la insuficiente incorporación de métodos de enseñanza-aprendizaje en el proceso (Nakova Katileva y Romero Carbonell, 2019).

En el contexto colombiano, se han gestado iniciativas y prácticas alrededor de la innovación educativa, mediada por la virtualidad. En este sentido, el 
Ministerio de Educación Nacional (MEN, 2013) se preocupó por las competencias TIC para el desarrollo profesional docente, con la finalidad de orientar programas de formación en los docentes en cinco competencias concretas: Competencia Tecnológica, Competencia Pedagógica, Competencia Comunicativa, Competencia de Gestión, y Competencia Investigativa.

En este mismo orden, el MEN (2016) advierte que es importante para los entornos de aprendizaje virtual considerar el diseño e implementación de recursos educativos integrados, articulados con los currículos que garanticen la incidencia y efectividad en los procesos de aprendizaje. Sin embargo, como lo expresa Aparicio Gómez (2019), además de ser competente en el uso pedagógico de las TIC, el docente ha de ser un sujeto comprometido, sensible y apasionado por su labor, ya que de él dependerá en gran medida la movilización y transformación de procesos educativos que incorporen estas herramientas. Lo cierto es que se ve un interés evidente por impulsar la producción y uso de recursos educativos digitales en el aula; por esta razón, la mayoría de los programas de formación en TIC pretenden que los docentes exploren y generen otras formas de enseñanza con tecnología.

En referencia a la profesión bibliotecológica puede mencionarse que se oficializa con la Ley 11 de 1979, en sus artículos 1 y 2, que reconocen los títulos de Bibliotecólogo o de Licenciado en Bibliotecología obtenidos en escuelas o facultades cuyos programas hayan sido aprobados por el MEN. El ámbito de aplicación de esta ley implica que los programas académicos deben denominarse de bibliotecología, de modo que la denominación propuesta para este programa se apoya en este referente legal y se justifica por la necesidad que existe a nivel nacional de contar con talento humano con altas competencias profesionales para gestionar adecuadamente todo tipo de bibliotecas y centros de documentación e información.

Con este programa se pretende cubrir la demanda de formación profesional en el área de bibliotecología en Colombia. En primer lugar, se requiere atender la formación profesional de la Red Nacional de Bibliotecas públicas, reconocida como la plataforma cultural más grande del país, con 1.540 servicios bibliotecarios distribuidos en los 32 departamentos que conforman la geografía nacional. También se pretende responder a las demandas de formación de alto nivel académico para cubrir el sector de bibliotecas universitarias, así como de las bibliotecas escolares de instituciones educativas públicas y privadas.

La Figura 1 presenta los espacios disciplinares que dialogarán desde la formación profesional, a saber: la bibliotecología y los estudios de información. 


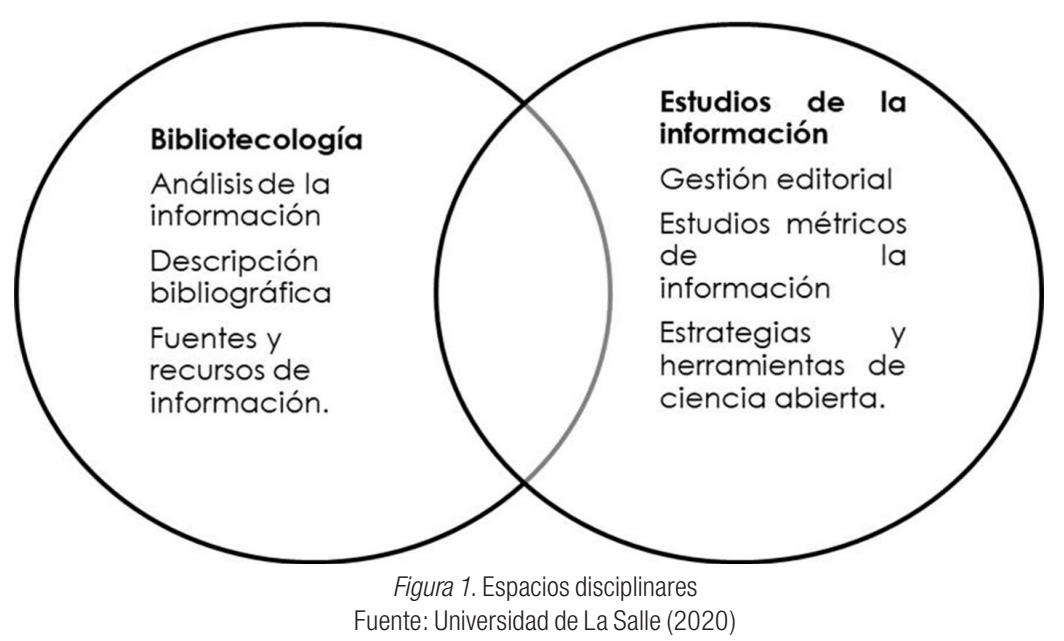

La denominación del Programa Virtual en Bibliotecología y Estudios de la Información de la Universidad de La Salle se apoya, en primer término, en las tendencias de la disciplina bibliotecológica en el contexto de los denominados estudios de información documental o Ciencias de la Información Documental, reconociendo el avance que ha tenido el campo no sólo en Colombia, sino en el mundo, en relación con la necesidad de orientar la formación hacia la gestión editorial, la medición de la información y el conocimiento científico y la organización y provisión de servicios articulados con estos entornos. En segundo término, este programa, aunque único en su tipo y enfoque en Colombia y en Iberoamérica, se fundamenta en la necesidad creciente en las instituciones de educación superior y centros e institutos de investigación de contar con profesionales que dialoguen con los gestores de políticas científicas, para cuya formulación, seguimiento y evaluación se requieren sistemas de gestión de la información científica, articulados desde los procesos de producción del conocimiento, hasta su organización y provisión de servicios y productos asociados.

En palabras de Morales Campos, Naranjo y Rendón Giraldo (2016), Colombia ha construido una importante trayectoria en la formación profesional de este campo; muestra de ello es la creación de la Escuela Interamericana de Bibliotecología de la Universidad de Antioquia en 1956, luego el Programa de Bibliotecología y Archivística de la Universidad de La Salle en 1971 y la organización y puesta en funcionamiento del Programa en Bibliotecología-Ciencia de la Información de la Pontificia Universidad Javeriana en 1973. Posterior a estas tres instituciones educativas que lideraron por muchos años 
en el país la formación profesional en bibliotecología, se crean otros programas académicos, como el de la Universidad del Quindío en 1987 y más recientemente el de la Fundación Universitaria INPAHU.

De tal manera que en Colombia hay una tradición de más de 60 años en la formación en este campo de conocimiento, convirtiéndose en referencia para la bibliotecología latinoamericana. Considerando el capital intelectual y formativo acumulado es que se presenta esta propuesta de Programa Virtual en Bibliotecología y Estudios de la Información, como un elemento que marca un diferencial por la novedad y proyección de futuro a la formación bibliotecológica, con un claro acento hacia la información científica, aquella que es producto de procesos de investigación rigurosa, sistemática y metódica para la superación de los problemas de la ciencia y la tecnología en diálogo con la sociedad.

Al revisar la Clasificación Internacional Normalizada de la Educación (CINE), planteada por la Unesco (2014), se encuentra claramente definido el campo de la Bibliotecología y la Información dentro de las Ciencias Sociales, Periodismo e Información, con lo cual se precisa la incorporación de este Programa Virtual en Bibliotecología y Estudios de la Información en el ámbito de la recién creada Escuela de Humanidades y Estudios Sociales en la Universidad de La Salle. Adscribir este programa en esta unidad académica permitirá el diálogo con áreas como la filosofía, la literatura, los estudios sociales y las artes.

El Marco Nacional de Cualificaciones (MNC), como referente nacional, asume que Colombia será la más educada de América Latina para 2025; este es un instrumento para clasificar por niveles de conocimientos, destrezas y actitudes de acuerdo con un conjunto de criterios sobre los aprendizajes logrados por las personas. Así, con el MNC se pretende promover el aprendizaje permanente, consolidando rutas de aprendizaje, mejorando el acceso, participación, progresión educativa y laboral de las personas.

El MNC permitirá articular y flexibilizar los sistemas de educación y de formación para dar respuestas oportunas y pertinentes a las demandas derivadas de la globalización y el desarrollo tecnológico a nivel mundial. En este sentido, se convierte en un instrumento articulador de las necesidades sociales con el mundo laboral, así como con los procesos educativos y formativos. El MNC aportará en la disminución de las brechas hoy existentes entre el sector productivo y académico, buscando fortalecer el capital humano del país a través de una oferta educativa y formativa pertinente y de calidad (MEN, 2016). 


\section{Método}

El objetivo planteado es diseñar la propuesta de un Programa Virtual en Bibliotecología y Estudios de la Información para la Universidad de La Salle de Bogotá-Colombia. El método se basó en un enfoque mixto, según el cual se amplían las posibilidades para afrontar la complejidad de los problemas de investigación planteados en todas las ciencias y enfocarlos de una manera holística. En este sentido, Hernández Sampieri, Fernández y Baptista (2010) plantean que la investigación mixta no tiene como meta reemplazar a la investigación cuantitativa ni a la cualitativa, sino utilizar las fortalezas de ambos tipos de indagación combinándolas y tratando de minimizar sus debilidades potenciales. Las técnicas fueron el análisis documental (comparación de programas académicos similares), la encuesta, mediante una muestra intencional-focalizada, y los lineamientos curriculares planteados por el Ministerio de Educación Nacional de Colombia y la Universidad de La Salle para los programas académicos en modalidad virtual.

El análisis documental partió de la revisión de los programas académicos similares, de acuerdo con la información disponible en fuentes oficiales e información web sobre los programas de Bibliotecología y Estudios de la Información, o Bibliotecología y Ciencia de la Información, o Información y Documentación de Argentina, Brasil, Bolivia, Chile, Colombia, Costa Rica, Cuba, Ecuador, Perú y España. La aplicación de esta técnica permitió el reconocimiento de las tendencias formativas proyectadas para este campo de conocimiento en Iberoamérica, lo que permitió a su vez identificar el valor agregado del programa virtual propuesto y las potencialidades para la construcción de redes de cooperación académica con instituciones de educación superior iberoamericanas.

La encuesta realizada mediante muestra intencional-focalizada permitió identificar a los estudiantes del Programa de Tecnología en Gestión Bibliotecaria del SENA (Sistema Nacional de Aprendizaje), entidad que a nivel nacional ofrece programas de formación tecnológica con dos años de duración, guiados por principios del modelo pedagógico de la educación para el trabajo. Se obtuvieron 98 respuestas, $86 \%$ correspondieron a estudiantes de esta institución y $14 \%$ no son egresados de esta institución. Los datos recolectados con la encuesta ofrecieron referentes de sustentación; desde la identificación de necesidades formativas en el ámbito nacional, esta propuesta formativa virtual ampliará la cobertura educativa más allá del territorio colombiano.

Finalmente, los lineamientos curriculares del Ministerio de Educación Nacional y de la Universidad de La Salle enfatizan que los principios metodológicos garanticen su efectividad en el aprendizaje, y para lograrlo se sigue 
el procedimiento establecido por el modelo de Análisis, Diseño, Desarrollo, Implementación y Evaluación (ADDIE), definido por el Ministerio de Educación Nacional como orientación para el diseño, producción e implementación de cursos virtuales.

\section{AnÁlisis De RESUltados}

La revisión de las ofertas formativas en el área de bibliotecología y estudios de la información reporta la presencia de núcleos disciplinares considerados como lo medular de la formación en este campo de conocimiento: organización de la información documental, fuentes, usuarios y servicios de información, tecnologías de información y comunicación, administración e investigación. Tales núcleos se consideran en la propuesta formativa virtual, pero se enfatizan tres componentes esenciales: la gestión editorial, los estudios métricos de la información, y las estrategias y técnicas de ciencia abierta. La apuesta que se realiza a partir de la revisión de ofertas similares es mantener lo esencial de la disciplina, pero incluyendo componentes considerados en estos momentos como estratégicos. De lo cual se propone la necesidad de un profesional de bibliotecología y estudios de la información con mayores condiciones habilitantes para dialogar con los ecosistemas científicos y la red de actores, infraestructuras, recursos y plataformas que lo componen.

Pasando a los resultados de la encuesta, se estudiaron indicadores como procedencia de los estudiantes y/o egresados del Programa de Tecnología en Gestión Bibliotecaria, principales inconvenientes para estudiar en la Universidad de La Salle y las preferencias en cuanto a la metodología para estudiar. La encuesta fue respondida por estudiantes del Programa Tecnológico en Gestión Bibliotecaria del Sistema Nacional de Aprendizaje de Colombia (SENA). En cuanto a la procedencia, la Figura 2 muestra la distribución de respuestas obtenidas según el departamento del país. La mayor parte de respuestas se concentraron en Bogotá, seguido por el Cesar, Norte de Santander y Cundinamarca, con lo cual se revela la procedencia de los posibles estudiantes del Programa Virtual en Bibliotecología y Estudios de la Información.

La Figura 3 muestra los inconvenientes expresados por los potenciales estudiantes del programa. Las cifras señalan cómo los dos problemas básicos son los económicos y los relacionados para radicarse en Bogotá para un programa presencial, lo que justifica que se estructuren los estudios en modalidad virtual. 


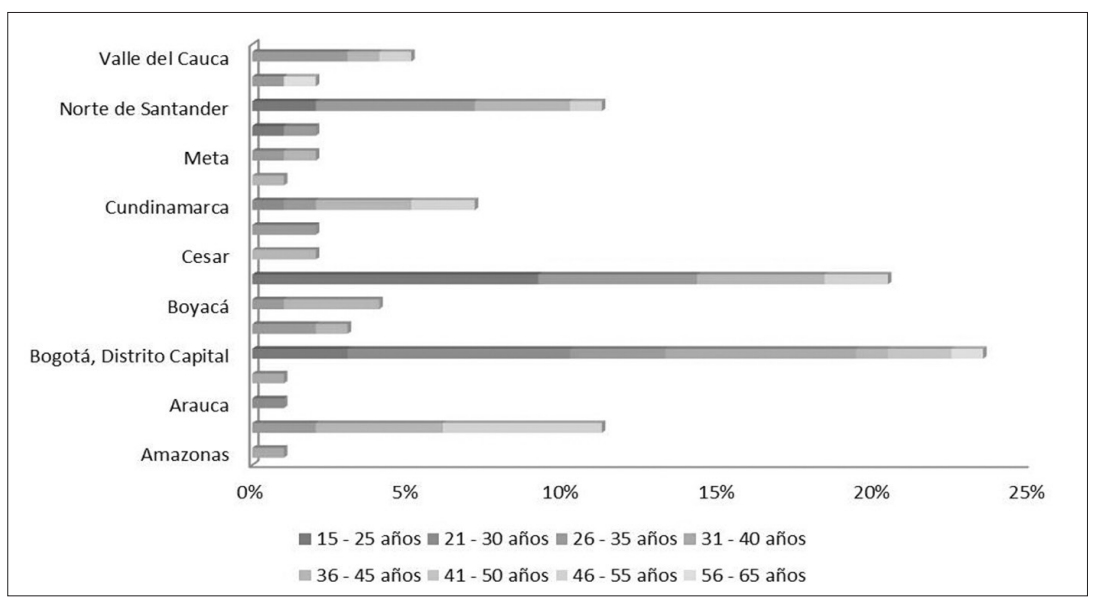

Figura 2. Departamento de procedencia de los informantes Fuente: elaboración propia

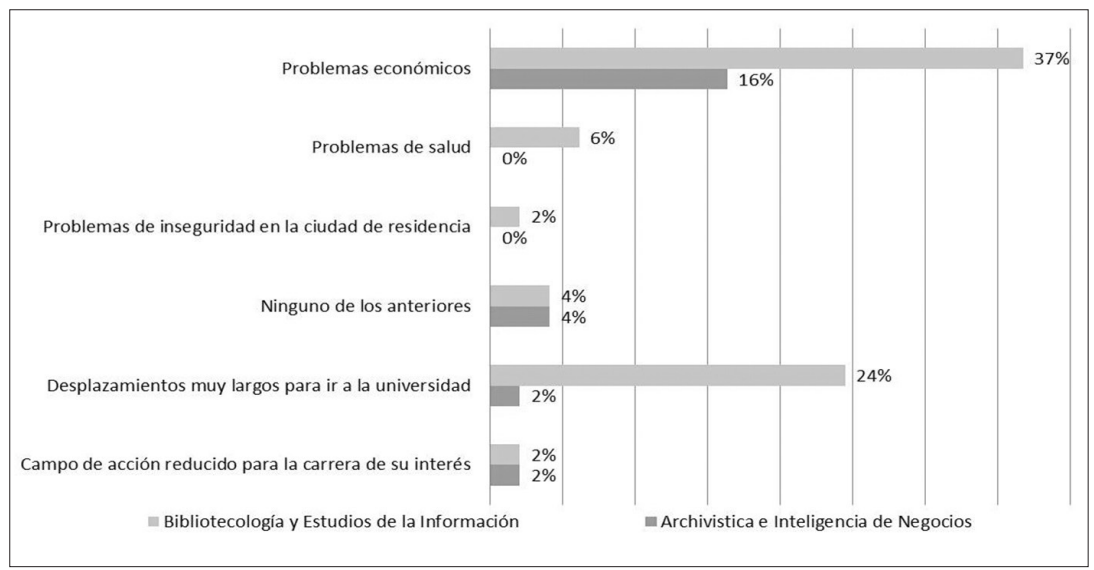

Figura 3. Problemas identificados para estudiar en el programa Fuente: elaboración propia

La Figura 4 señala el alto porcentaje de respuestas obtenidas por los estudiantes potenciales del programa en cuanto a la planeación de estudiar bibliotecología. El 59 \% expresó la importancia de la modalidad virtual, frente a $8 \%$ que señaló como modalidad la semipresencial, $6 \%$ a distancia y $2 \%$ presencial. Al cruzar todos los resultados de la aplicación de la encuesta, se constata la alta potencialidad que tiene un Programa Virtual en Bibliotecología y Estudios de la Información en la Universidad de La Salle. 


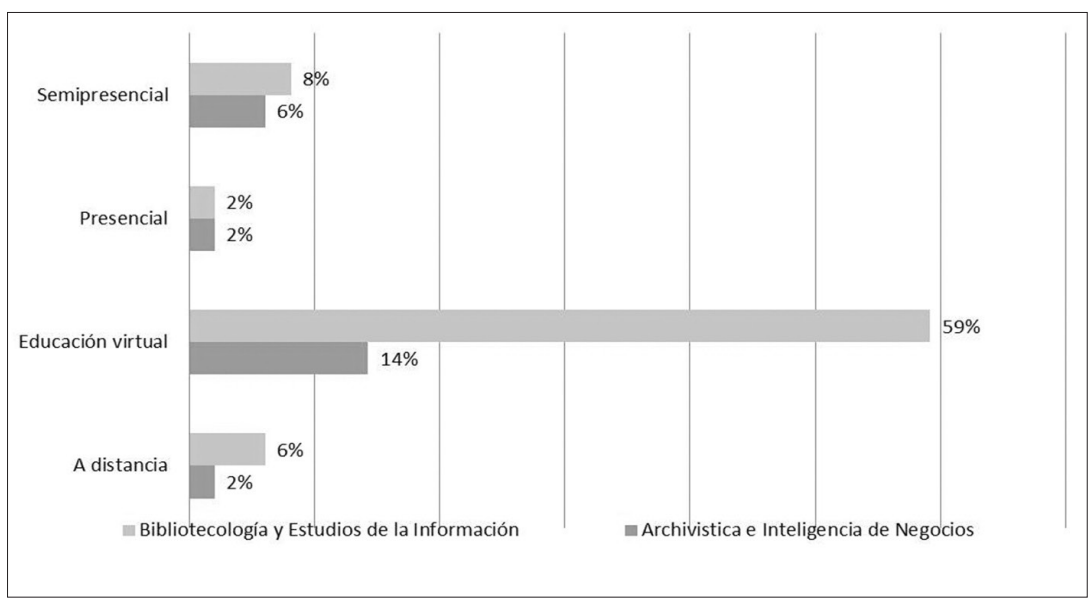

Figura 4. Metodología en la que se prefiere estudiar

Fuente: elaboración propia

En cuanto a los lineamientos curriculares expresados tanto por el Ministerio de Educación Nacional de Colombia como por la Universidad de La Salle para el diseño y gestión de programas académicos virtuales, como eje fundamental del proceso formativo en los escenarios virtuales, profundizan en los contenidos propuestos en los encuentros sincrónicos y retroalimentan el proceso de manera permanente (Universidad de La Salle, 2008: 15). De igual manera, y en armonía con el Proyecto Educativo Universitario Lasallista (PEUL), se sustenta esta perspectiva, sobre todo en la promoción por parte del docente de diálogos permanentes con las pedagogías contemporáneas y la "búsqueda de la equidad", en procura de democratizar el conocimiento (Universidad de La Salle, 2007: 11).

Los principios se traducen en estrategias didácticas para el desarrollo de los espacios académicos virtuales en la Universidad de La Salle como pilares del componente pedagógico en el modelo educativo, siendo allí donde se materializa el proceso de aprendizaje. Tomando como base la perspectiva educativa virtual para la universidad, se proponen estrategias de enseñanza y aprendizaje con las bases pedagógicas y didácticas para la virtualidad, que expone el referente pedagógico internacional "SMART" (KERIS, 2012). Este referente se utiliza como guía para la construcción de escenarios educativos innovadores para la educación virtual en diferentes proyectos educativos nacionales e internacionales, como en el caso del proyecto nacional "Construyendo capacidades en uso de TIC para innovar en educación”, liderado por el MEN (2013). 


\section{Propuesta de Programa Virtual en Bibliotecología y ESTUdios DE LA INFORMACIÓN}

El diseño de una propuesta para el nuevo Programa Virtual en Bibliotecología y Estudios de la Información es el resultado concreto de la integración de los resultados del ejercicio de investigación-innovación curricular, desarrollado sobre la base de las técnicas utilizadas, en el marco de las transformaciones académico-administrativas que se están dando en la Universidad de La Salle como parte de la puesta en marcha de nuevas unidades académicas y programas formativos. En este sentido, se presenta a continuación el perfil de competencias, estructura de la malla curricular y matriz de resultados de aprendizaje. Cabe destacar que el diseño curricular por resultados de aprendizaje se acoge a lo planteado en la Resolución 021795 de 2020, emitida por el Ministerio de Educación Nacional de Colombia para la obtención, modificación y renovación del registro calificado de los programas académicos en el país.

\section{Perfiles integrales expresados en competencias (perfil de egreso)}

Las competencias que se desarrollarán a partir de la formación en el Programa Virtual de Bibliotecología y Estudios de la Información son las siguientes:

1. Organiza información, según estándares internacionales, proyectando planes y programas de formación de usuarios para la maximización de los servicios-productos que promuevan la aplicación del conocimiento en ámbitos académicos y profesionales, sobre la base de los principios y criterios de la ciencia abierta.

2. Gestiona escenarios editoriales, aplicando estrategias y normativas de ciencia abierta para el logro de la visibilidad y posicionamiento estratégico de la información científica, en perspectiva de los postulados de la apertura del conocimiento científico para impulsar entornos de apropiación social de la ciencia.

3. Desarrolla estudios métricos de información para la generación de los insumos necesarios que permitan la formulación, seguimiento y evaluación de políticas de ciencia y tecnología en diferentes campos de conocimiento, a partir de indicadores.

\section{Estructura de la malla curricular}

El Programa Virtual de Bibliotecología y Estudios de la Información tiene 140 créditos académicos, distribuidos en ocho semestres (Consejo Superior, 
No. 002 de enero de 2021) y cinco áreas de la estructura curricular, como se muestra en la Tabla 1.

\begin{tabular}{|c|c|c|c|}
\hline Área & Espacios académicos & No. de créditos & $\%$ \\
\hline \multirow[t]{8}{*}{ Fundamentación } & Fundamentos de Bibliotecología & \multirow[t]{8}{*}{27} & \multirow[t]{8}{*}{19,29} \\
\hline & Fundamentos de Información Científica & & \\
\hline & Epistemología de la Bibliotecología & & \\
\hline & Gestión Editorial I & & \\
\hline & Gestión Editorial II & & \\
\hline & Estudios Métricos I & & \\
\hline & Estudios Métricos II & & \\
\hline & Bibliotecas Digitales & & \\
\hline \multirow[t]{21}{*}{ Profesional } & Clasificación de las Ciencias & \multirow[t]{21}{*}{69} & \multirow[t]{21}{*}{49,29} \\
\hline & Introducción al Análisis de la Información & & \\
\hline & Descripción Bibliográfica & & \\
\hline & Fuentes y Recursos de la Información I & & \\
\hline & Fuentes y Recursos de la Información II & & \\
\hline & Desarrollo de Colecciones & & \\
\hline & Lenguajes Documentales & & \\
\hline & Pedagogía y Didáctica aplicada a la Bibliotecología & & \\
\hline & Bibliotecas Universitarias & & \\
\hline & Bibliotecas Públicas y Escolares & & \\
\hline & Estrategias y Herramientas de Ciencia Abierta & & \\
\hline & Políticas de Ciencia y Tecnología & & \\
\hline & Mercadeo de la Información & & \\
\hline & |nglés | & & \\
\hline & Inglés II & & \\
\hline & Proyecto Integrador I & & \\
\hline & Proyecto Integrador II & & \\
\hline & Proyecto Integrador III & & \\
\hline & Emprendimiento & & \\
\hline & Formulación y Evaluación de Proyectos & & \\
\hline & Políticas de Ciencia y Tecnología & & \\
\hline
\end{tabular}




\begin{tabular}{|c|c|c|c|}
\hline \multirow{6}{*}{$\begin{array}{l}\text { Fundamentación } \\
\text { investigativa }\end{array}$} & Habilidades Básicas I & \multirow[t]{6}{*}{16} & \multirow[t]{6}{*}{11,43} \\
\hline & Habilidades Básicas II & & \\
\hline & Habilidades Investigativas I & & \\
\hline & Habilidades Investigativas II & & \\
\hline & Modalidad de Grado I & & \\
\hline & Modalidad de Grado II & & \\
\hline \multirow{4}{*}{$\begin{array}{l}\text { Formación } \\
\text { Lasallista }\end{array}$} & Identidad Lasallista & \multirow[t]{4}{*}{10} & \multirow[t]{4}{*}{7,14} \\
\hline & Humanidades & & \\
\hline & Cultura Religiosa I y II & & \\
\hline & Ética & & \\
\hline \multirow[t]{7}{*}{ Formación Electiva } & Electiva I & \multirow[t]{6}{*}{18} & \multirow[t]{6}{*}{12,86} \\
\hline & Electiva II & & \\
\hline & Electiva III & & \\
\hline & Electiva IV & & \\
\hline & Electiva V & & \\
\hline & Electiva VI & & \\
\hline & & 140 & $100 \%$ \\
\hline
\end{tabular}

Tabla 1. Descripción de las áreas de formación y articulación con espacios académicos Fuente: Consejo Superior, Acuerdo No. 002 de enero de 2021

\section{Resutados de aprendizaje}

Los resultados de aprendizaje permiten precisar los logros finales que los estudiantes deben alcanzar como producto de su proceso formativo. Los resultados especifican aún más las competencias y traducen las formas concretas mediante las cuales se pueden evidenciar, mediante acciones observables, el conjunto de indicadores que permiten dilucidar si se desarrolló la competencia. La Tabla 2 muestra la relación entre las competencias y cada uno de los resultados de aprendizaje. 


\section{Resultados de aprendizaje}

1.1. Analiza los fundamentos de la bibliotecología considerando elementos teóricos y epistemológicos. 1.2. Caracteriza la organización de información y conocimiento como subcampo fundamental de las ciencias de la información documental.

1.3. Establece los tipos de análisis de información para aplicarlos a la organización de la información científica.

1.4. Diferencia el análisis formal del análisis documental de contenido.

1.5. Realiza procesos de descripción bibliográfica según estándares y normativa.

1.6. Diseña políticas de desarrollo de colecciones impresas y digitales como componente esencial de la gestión de unidades y servicios de información.

1.7. Aplica estrategias para la búsqueda y recuperación avanzada de información científica disponible en fuentes y recursos.

1.8. Aplica criterios para la evaluación y selección de fuentes de información científica.

1.9. Desarrolla estudios de usuarios para el diseño de planes de mercadeo de la información.

1.10. Posiciona estratégicamente productos y servicios de información científica mediante análisis de variables del mercado.

2.1. Aplica los procesos esenciales de la gestión editorial en la perspectiva de la ciencia abierta y visibilidad de la información científica.

2.2. Desarrolla modelos de evaluación de revistas científicas, atendiendo a criterios de ciencia abierta y visibilidad de la información científica.

2.3. Aplica estándares y normativa para el diseño de repositorios y bibliotecas digitales.

2.4. Evalúa repositorios digitales institucionales de información científica.

2.5. Gestiona recursos y procesos en Bibliotecas Universitarias, articulados con la legislación y políticas académicas de las instituciones de educación superior.

2.6. Gestiona recursos y procesos en Bibliotecas Públicas y Escolares, articulados con la legislación y políticas educativas, de lectura y bibliotecas.

2.7. Diseña políticas y estrategias de ciencia abierta y visibilidad de la información científica en instituciones de educación superior e institutos de investigación.

2.8. Evalúa modelos de ciencia abierta según estándares internacionales, normatividad y criterios nacionales e institucionales.

2.9. Lidera la construcción de contenidos en repositorios digitales de información científica.

3.1. Analiza los fundamentos de la información científica, visualizando la importancia de los estudios métricos.

3.2. Perfila los estudios métricos de la información como conjunto de conocimientos, metodologías y herramientas de evaluación del comportamiento de la ciencia.

3.3. Identifica las disciplinas que conforman las especialidades métricas de la información, reconociendo la naturaleza y alcance de cada una.

3.4. Establece relaciones entre comunicación científica y estudios métricos de la información.

3.5. Aplica las leyes bibliométricas para la evaluación de la información científica.

3.6. Aplica indicadores métricos en la evaluación de diferentes campos del conocimiento científico.

3.7. Contextualiza resultados de los estudios métricos de información y los aplica al mejoramiento de la gestión de unidades y servicios de información.

3.8. Evalúa la producción y uso de información científica en conexión con políticas de ciencia y tecnología.

3.9. Participa activamente en la formulación, implementación y evaluación de políticas de ciencia y tecnología.

3.10. Diseña lenguajes documentales para la representación, normalización y recuperación de información científica.

3.11. Diseña y ejecuta proyectos integradores para la medición y gestión integral de la información científica.

Tabla 2. Matriz de resultados de aprendizaje esperados 


\section{Conclusiones}

Se plantea la necesidad de ofrecer programas académicos en el campo de la bibliotecología y los estudios de la información, bajo la modalidad virtual, para ampliar la oferta formativa que otorgue mayores posibilidades de inclusión social tan necesaria no sólo en el ámbito colombiano, sino en América Latina de manera general, desde la apertura que plantea la ciencia abierta para romper con las restricciones a los contenidos académicos.

Estos planteamientos abren un abanico de posibilidades para la labor bibliotecológica, para lo cual es necesario desarrollar competencias concretas para la intervención del conocimiento que haga posible también la participación y aportes de los profesionales en la creación de innovadores contenidos científicos a partir de la interacción con contenidos abiertos y de libre acceso, que a su vez den paso a nuevos análisis, productos y servicios ajustados a las necesidades sociales. Se trata del establecimiento de un diálogo entre la bibliotecología y la ciencia en una visión horizontal y se muestre una implementación real de la información para la solución de problemática y sociales.

La propuesta formativa será de gran aporte para la Red Nacional de Bibliotecas Públicas de Colombia, así como para cubrir las demandas de alto nivel desde las bibliotecas universitarias, escolares, de instituciones educativas de los sectores tanto público como privado, principalmente por la modalidad virtual en términos de responder a una de las necesidades básicas arrojadas durante el estudio, la cual se relaciona con las distancias largas que deben recorrer los estudiantes para formarse, además de poder atender a los interesados de todos los municipios de Colombia y otras latitudes.

Finalmente se muestra un perfil basado en tres competencias integrales, la malla curricular constituida por 140 créditos académicos establecidos en función de cinco áreas de formación, con una duración de ocho periodos semestrales. Una vez concluida la formación se espera que los aprendizajes adquiridos sean evidenciados en las prácticas de desempeño profesional en función de los indicadores establecidos por competencias.

\section{REFERENCIAS}

Anglada, Lluís y Ernest Abadal. 2018. “¿Qué es la ciencia abierta?”. Anuario ThinkEPI (12): 292-298.

https://doi.org/10.3145/thinkepi.2018.43

Aparicio Gómez, Oscar Yecid. 2019. "El uso educativo de las TIC". Revista Interamericana de investigación, educación y pedagogía 12 (1): 211-227. https://revistas.usantotomas.edu.co/index.php/riiep/article/view/4904/4617 
Hernández Sampieri, Roberto, Carlos Fernández y Pilar Baptista. 2010. Metodología de la Investigación. México: McGraw Hill.

KERIS (Korea Education and Research Information Service). 2012. Adapting Education to the Information Age.

https://asemlllhub.org/wp-content/uploads/attachments/Republic_of_Korea_-_ Adapting_education_to_the_information_age.pdf

MEN (Ministerio de Educación Nacional). 1979. Ley 11 de 1979. https://minciencias.gov.co/sites/default/files/upload/reglamentacion/ley-11-1979.pdf

MEN. 2013. Competencias TIC para el profesional docente. https:/www.mineducacion.gov.co/1759/articles-339097_archivo_pdf_competencias_tic.pdf

MEN. 2016. Marco Nacional de Cualificaciones. https://www.mineducacion.gov.co/1759/w3-propertyvalue-58999.html?_noredirect $=1$

MEN. 2020. Resolución 021795. https://www.mineducacion.gov.co/1759/articles-402045_pdf.pdf

Merchán, Carlos. 2018. "Modelamiento pedagógico de Ambientes Virtuales de Aprendizaje (AVA)”. TED 44 (2): 51-70.

https://revistas.pedagogica.edu.co/index.php/TED/article/view/8989

Morales Campos, Estela, Edilma Naranjo y Nora Rendón Giraldo. 2016. Forjadores e impulsores de la Bibliotecología Latinoamericana. Colombia. México: Instituto de Investigaciones Bibliotecológicas, Universidad Nacional Autónoma de México. http://ru.iibi.unam.mx/jspui/handle/IIBI_UNAM/L91

Morales Salas, Rubí, Juan Carlos Infante Moro y Julia Gallardo Pérez. 2019. "La mediación e interacción en un AVA para la gestión eficaz en el aprendizaje virtual". Campus Virtuales 8 (1): 49-61.

http://uajournals.com/ojs/index.php/campusvirtuales/article/view/425

Nakova Katileva, Eleonora y Marc Romero Carbonell. 2019. "Percepciones sobre la influencia de los estilos de aprendizaje en el aprendizaje colaborativo en entornos virtuales". Edutec. Revista Electrónica de Tecnología Educativa (69): 23-35.

https://www.edutec.es/revista/index.php/edutec-e/article/view/1391

Peláez Sanpedro, Marcia. 2019. “Diseño y validación de la propuesta educativa RED en el Aula, orientada a fortalecer desde el enfoque del Aprendizaje Significativo la Competencia Tecnológica en estudiantes de tercer semestre de Licenciatura en Comunicación e Informática Educativas de la Universidad Tecnológica de Pereira, por medio de la incorporación de un entorno de aprendizaje virtual para la producción e integración de recursos educativos digitales (RED) en el aula”. Tesis de maestría, Universidad Tecnológica de Pereira-Colombia, Facultad de Ciencias de la Educación.

http://repositorio.utp.edu.co/dspace/bitstream/handle/11059/11757/T378.173466\%20

P382.pdf?sequence $=1$ \&isAllowed $=\mathrm{y}$

Ramírez, Paola Andrea y Daniel Samoilovich. 2019. "La promesa de la ciencia abierta". Revista de Educación Superior ESAL (5).

https://rcientificas.uninorte.edu.co/index.php/esal/article/view/11804

Rozemblum, Cecilia. 2019. AmeliCA. Encontro Nacional de Portais de Periodicos 2, 26 slides. https://econtents.bc.unicamp.br/eventos/index.php/enapp/article/view/1555

Unesco. 2014. Clasificación Internacional Normalizada de la Educación. http://uis.unesco.org/sites/default/files/documents/isced-2011-sp.pdf 
Unesco. 2021. Anteproyecto de recomendación de la Unesco sobre la ciencia abierta. https://es.unesco.org/sites/default/files/es-20-03117_anteproyecto_de_recomendacion_de_la_unesco_sobre_la_ciencia_abierta.pdf

Universidad de La Salle. 2007. Proyecto Educativo Universitario Lasallista (PEUL). Bogotá-Colombia. http://ciencia.lasalle.edu.co/librillos/24

Universidad de La Salle. 2008. Enfoque Formativo Lasallista (EFL). Bogotá-Colombia. http://ciencia.lasalle.edu.co/librillos/29

Universidad de La Salle. 2020. Documento maestro del Programa de Bibliotecología y Estudios Sociales. Bogotá-Colombia: Facultad de Ciencias Económicas y Sociales.

Para citar este texto:

Pirela Morillo, Johann Enrique, Luis Fernando Sierra Escobar y Yamely Almarza Franco. 2022. "Propuesta de un Programa Virtual en Bibliotecología y Estudios de la Información para la Universidad de La Salle, Bogotá-Colombia”. Investigación Bibliotecológica: archivonomía, bibliotecología e información 36 (90): 67-85.

http://dx.doi.org/10.22201/iibi.24488321xe.2022.90.58460 